\title{
Standardization of evaluation method and prognostic significance of histological response to preoperative chemotherapy in high-grade non-round cell soft tissue
} sarcomas

Yoshinao Oda ${ }^{1}$, Kazuhiro Tanaka ${ }^{2}$, Takanori Hirose ${ }^{3}$, Tadashi Hasegawa ${ }^{4}$, Nobuyuki Hiruta ${ }^{5}$, Masanori Hisaoka ${ }^{6}$, Masato Yoshimoto ${ }^{1}$, Hiroshi Otsuka' ${ }^{1}$, Hirofumi Bekki ${ }^{1}$, Takeaki Ishii ${ }^{1}$, Makoto Endo $^{7}$, Toshiyuki Kunisada ${ }^{8}$, Toru Hiruma ${ }^{9}$, Hiroyuki Tsuchiya ${ }^{10}$, Hirohisa Katagiri ${ }^{11}$, Yoshihiro Matsumoto ${ }^{7}$, Akira Kawai ${ }^{12}$, Robert Nakayama ${ }^{13}$, Hiroyuki Kawashima ${ }^{14}$, Satoshi Takenaka ${ }^{15}$, Makoto Emori ${ }^{16}$, Munenori Watanuki ${ }^{17}$, Yukihiro Yoshida ${ }^{18}$, Takeshi Okamoto ${ }^{19}$, Junki Mizusawa ${ }^{20}$, Haruhiko Fukuda ${ }^{20}$, Toshifumi Ozaki21, Yukihide Iwamoto ${ }^{22^{*}}$ and Takayuki Nojima ${ }^{23}$

\begin{abstract}
Background: Preoperative chemotherapy is widely applied to high-grade localized soft tissue sarcomas (STSs); however, the prognostic significance of histological response to chemotherapy remains controversial. This study aimed to standardize evaluation method of histological response to chemotherapy with high agreement score among pathologists, and to establish a cut-off value closely related to prognosis.

Methods: Using data and specimens from the patients who had registered in the Japan Clinical Oncology Group study, JCOG0304, a phase II trial evaluating the efficacy of perioperative chemotherapy with doxorubicin (DOX) and ifosfamide (IFO), we evaluated histological response to preoperative chemotherapy at the central review board.

Results: A total of 64 patients were eligible for this study. The percentage of viable tumor area ranged from $0.1 \%$ to $97.0 \%$, with median value of $35.7 \%$. Regarding concordance proportion between pathologists, the weighted kappa coefficient $(k)$ score in all patients was 0.71 , indicating that the established evaluation method achieved substantial agreement score. When the cut-off value of the percentage of the residual tumor area was set as $25 \%$, the $p$-value for the difference in overall survival showed the minimum value. Hazard ratio of the non-responder with percentage of the residual tumor $<25 \%$, to the responder was 4.029 (95\% confidence interval $0.893-18.188, p=0.070$ ).
\end{abstract}

Conclusion: The standardized evaluation method of pathological response to preoperative chemotherapy showed a substantial agreement in the weighted $k$ score. The evaluation method established here was useful for estimating of the prognosis in STS patients who were administered perioperative chemotherapy with DOX and IFO.

\footnotetext{
*Correspondence: yiwamoto@kyushuh.johas.go.jp

${ }^{22}$ Department of Orthopaedic Surgery, Kyushu Rosai Hospital,

Kitakyushu, Japan

Full list of author information is available at the end of the article
}

(C) The Author(s) 2022. Open Access This article is licensed under a Creative Commons Attribution 4.0 International License, which permits use, sharing, adaptation, distribution and reproduction in any medium or format, as long as you give appropriate credit to the original author(s) and the source, provide a link to the Creative Commons licence, and indicate if changes were made. The images or other third party material in this article are included in the article's Creative Commons licence, unless indicated otherwise in a credit line to the material. If material is not included in the article's Creative Commons licence and your intended use is not permitted by statutory regulation or exceeds the permitted use, you will need to obtain permission directly from the copyright holder. To view a copy of this licence, visit http://creativecommons.org/licenses/by/4.0/. The Creative Commons Public Domain Dedication waiver (http://creativeco mmons.org/publicdomain/zero/1.0/) applies to the data made available in this article, unless otherwise stated in a credit line to the data. 
Trial registration: UMIN Clinical Trials Registry C000000096. Registered 30 August, 2005 (retrospectively registered).

Keywords: Histological response, Chemotherapy, Doxorubicin, Ifosfamide, Soft tissue sarcoma, Clinical trial, Prognosis, Neoadjuvant

\section{Background}

Soft tissue sarcomas (STS) are a heterogenous group of rare malignant tumors with a wide spectrum in terms of histological findings, which comprise less than $1 \%$ of all malignant tumors [1]. The standard treatment of STS is based on the clinical stage of the tumor. The American Joint Committee on Cancer (AJCC)/International Union against Cancer (UICC) staging system is the most widely used for the staging [2]. Preoperative chemotherapy has been reported to be effective for localized high-risk STS [3]. The current standard preoperative chemotherapy for STS is anthracyclin-containing regimen including a combination of doxorubicin (DOX) and ifosfamide (IFO) is widely accepted [4-7].

Even when chemotherapy is effective, STS is not always reduced, as the tumor diameter sometimes gets enlarged because of the expansion in tumor mass due to intratumoral hemorrhage, necrosis, edema, fibrosis, and hyalinization caused by antitumor agents. A correlation between changes in tumor size on the radiological images and patient prognosis is controversial $[8,9]$. Similarly, the prognostic significance of histological response to preoperative chemotherapy in high-grade STS has not been established yet. Some studies have reported a positive association between prognosis and histological response, whereas others have reported a negative association [10-18]. Therefore, there is a growing interest in the evaluation of histological effects in an attempt to assess prognosis after chemotherapy.

One of the problems is that a detailed standard evaluation method of histological response after chemotherapy on STS has not been established; therefore, differences in judgment among pathologists are likely to occur. There is no specific definition for determining whether tumor cells are viable or non-viable. The tumor cells show a wide variety of degenerative histological findings including pyknosis, vacuolation, hypertrophy and karyorrhexis, or eosinophilic change of cytoplasm. Evaluating viability of the degenerated tumor cells is often difficult, and thus it is important to define strict evaluation criteria for this purpose.

Another problem is that each paper has adopted different cut-off values to analyze the association of specific factors, such as necrosis or residual tumor, with prognosis. Some papers defined a cut-off value as $95 \%$ of necrotic proportion, others used that of $75 \%$ necrotic proportion or that of $50 \%$ residual tumor proportion. No standard cut-off value to assess the prognostic effect of histological response to chemotherapy has been established yet. In addition, information on preoperative treatment such as the type of drugs used or dose administered varies widely even within a single study. Furthermore, it was not clear whether combining radiotherapy or regional hyperthermia with chemotherapy were different among different studies conducted on the preoperative treatment because almost all previous studies were retrospective in nature.

Therefore, the aims of this study were to establish definite criteria for evaluating histological response to chemotherapy, which has high concordance rate among pathologists, and to determine a cut-off value for the percentage of residual viable tumor cells, which shows the smallest $\mathrm{p}$ value for the difference in patient survival.

\section{Patients and Methods}

\section{Ethical statement.}

All methods were carried out in accordance with relevant guidelines and regulations. This study (the Japan Clinical Oncology Group study, JCOG0304-A1) protocol was approved by the Protocol Review Committee of the JCOG and by the Institutional Review Boards in each of the 20 participating institutes.

\section{Patients}

JCOG0304A1 is an accompanying research of a phase II trial evaluating the efficacy of perioperative chemotherapy with DOX and IFO for localized high-grade STSs in the extremities (JCOG0304) [6, 7]. All patient data and specimens used in this study were obtained from the patients who had registered in JCOG0304. JCOG0304 was conducted by the Bone and Soft Tissue Tumor Study Group of the JCOG. The details of eligibility criteria of JCOG0304 have been published previously $[6,7,19]$. The major inclusion criteria of the trial were as follows: (1) A histological diagnosis of non-round cell STSs including undifferentiated pleomorphic sarcoma (malignant fibrous histiocytoma), fibrosarcoma, leiomyosarcoma, synovial sarcoma, liposarcoma, pleomorphic rhabdomyosarcoma or undifferentiated sarcoma using open biopsy specimen; (2) FNCLCC histological grading system [20]: Grade 2 or 3; (3) AJCC stage (the 6th edition) [21]: Stage III (T2bNOM0); (4) resectable localized tumor in the extremities; (5) no history of treatment for non-round cell STS; (6), no history of chemotherapy or radiation therapy for any cancer; (7) age between 20 
and 70 years; (8) written informed consent. The patients were treated by preoperative chemotherapy with DOX $\left(30 \mathrm{mg} / \mathrm{m}^{2} /\right.$ day, days 1 and 2$)$ and IFO $\left(2 \mathrm{~g} / \mathrm{m}^{2} /\right.$ day, days 1 to 5), which was repeated three times every 3 weeks. Preoperative radiotherapy was not allowed in JCOG0304. The tumor was resected within 5 weeks from the last cycle of preoperative chemotherapy. Postoperatively, two cycles of DOX and IFO were carried out at intervals of 3 weeks.

Among the patients enrolled in JCOG0304 study, those whose pathological specimens of the maximum crosssectional slice of the tumor were available after receiving at least one cycle of preoperative chemotherapy were included in this accompanying study. of the resected tumor (Fig. 1). The cellularity of the residual viable cells compared to preoperative biopsy specimen was also considered. As a concrete procedure, the pathologists reviewed the virtual macroscopic slides and determined the extent of the viable area, and then marked them in the printed macroscopic picture of the cut-surface of the tumor. In addition, the tumor bed, in which the tumor was likely to have been present prior to preoperative chemotherapy was marked. If the viable cells were found reduced in cellularity due to chemotherapy, a biopsy specimen was used as a reference. The cellularity of residual viable tumor cells was classified into four categories: equivalent to $100 \%$, $50 \%, 25 \%$, and $5 \%$, respectively, compared to the cellularity in the biopsy specimen. Finally, the percentage of residual tumor was calculated as follows:

$\left[100 \%\right.$ viabletumorarea $\left.\left(\mathrm{cm}^{2}\right)\right]+\left[50 \%\right.$ viablearea $\left.\left(\mathrm{cm}^{2}\right)\right] \times 0.5+\left[25 \%\right.$ viablearea $\left.\left(\mathrm{cm}^{2}\right)\right] \times 0.25+\left[5 \%\right.$ viablearea $\left.\left(\mathrm{cm}^{2}\right)\right] \times 0.05$

[theareaoftumorbed $\left.\left(\mathrm{cm}^{2}\right)\right]$

\section{Radiological evaluation}

In JCOG0304, the radiological response to preoperative chemotherapy was evaluated using magnetic resonance imaging (MRI) [6]. The radiological response was centrally reviewed and assessed according to several kinds of criteria, including the WHO criteria [22]. Briefly, in the WHO criteria, the product of the largest perpendicular diameters on the cross section of the tumor was calculated, and the responses were judged as follows: complete response (CR), no residual tumor; partial response (PR), $50 \%$ or greater decrease; stable disease (SD), less than $50 \%$ decrease or less than $25 \%$ increase; progressive disease (PD), $25 \%$ or greater increase.

\section{Histological evaluation}

In JCOG0304A1, we evaluated histological response by evaluating the area and cellularity of the residual viable tumor cells. STSs showed a variety of histological findings when responded to chemotherapy. They included hyalinization, fibrosis, cystic change, foreign body reaction, and an aggregation of foamy cells, in addition to necrosis. We decided to count the residual viable tumor area but not necrotic area to evaluate the histological response to chemotherapy because discriminating between viable and degenerative area was considered easier than distinguishing between necrotic and degenerative area.

Viable and non-viable tumor cells were defined as follows: tumor cells showing cellular swelling, nuclear swelling, increased eosinophilia of cytoplasm, slight vacuolation were considered viable, whereas those showing any evidence of pyknosis, karyorrhexis, karyolysis, severe vacuolation, or loss of nuclear staining were considered non-viable.

The percentage of viable tumor area was calculated by the area in which viable tumor cells survived divided by the area of the whole tumor bed on the largest cut-surface
The percentage of residual tumor area was calculated as a continuous variable and used in the statistical analysis later.

\section{Central pathology review}

First, six pathologists specialized in STS (YO, THiro, $\mathrm{THa}, \mathrm{NH}, \mathrm{MH}$, and $\mathrm{TN}$ ) discussed and determined the criteria for evaluation of pathological findings to assess the residual viable tumor cells after chemotherapy. Thereafter, 10 cases for test set (Group A) were randomly selected from the collected specimens, and each pathologist independently reviewed and scored the Group A cases. At the central pathology review, the pathologists discussed the histological findings causing mismatches according to the concordance rate of each case. Next, each pathologist independently reviewed 10 cases for the first validation set (Group B), which were randomly chosen from the remaining cases excluding those in Group A. When the concordance rate of Group B exceeded that of Group A and exceeded the minimum limit value of the concordance rate (weighted kappa coefficient $[k]>0.6$ ), the criteria for assessment were finalized and used to evaluate the remaining cases (Group C).

\section{Statistical analyses}

With regard to case selection in each group, stratified random sampling was conducted according to histology (polymorphic cell sarcoma vs others) and histological grade evaluated by institutions (grade 0,1 vs. 2, 3). As a measure of agreement, $k$ with multiple evaluaters [23, 24] in each pair of the six pathologists, i.e., 15 combinations, was calculated for the percentage of residual tumor cells. 


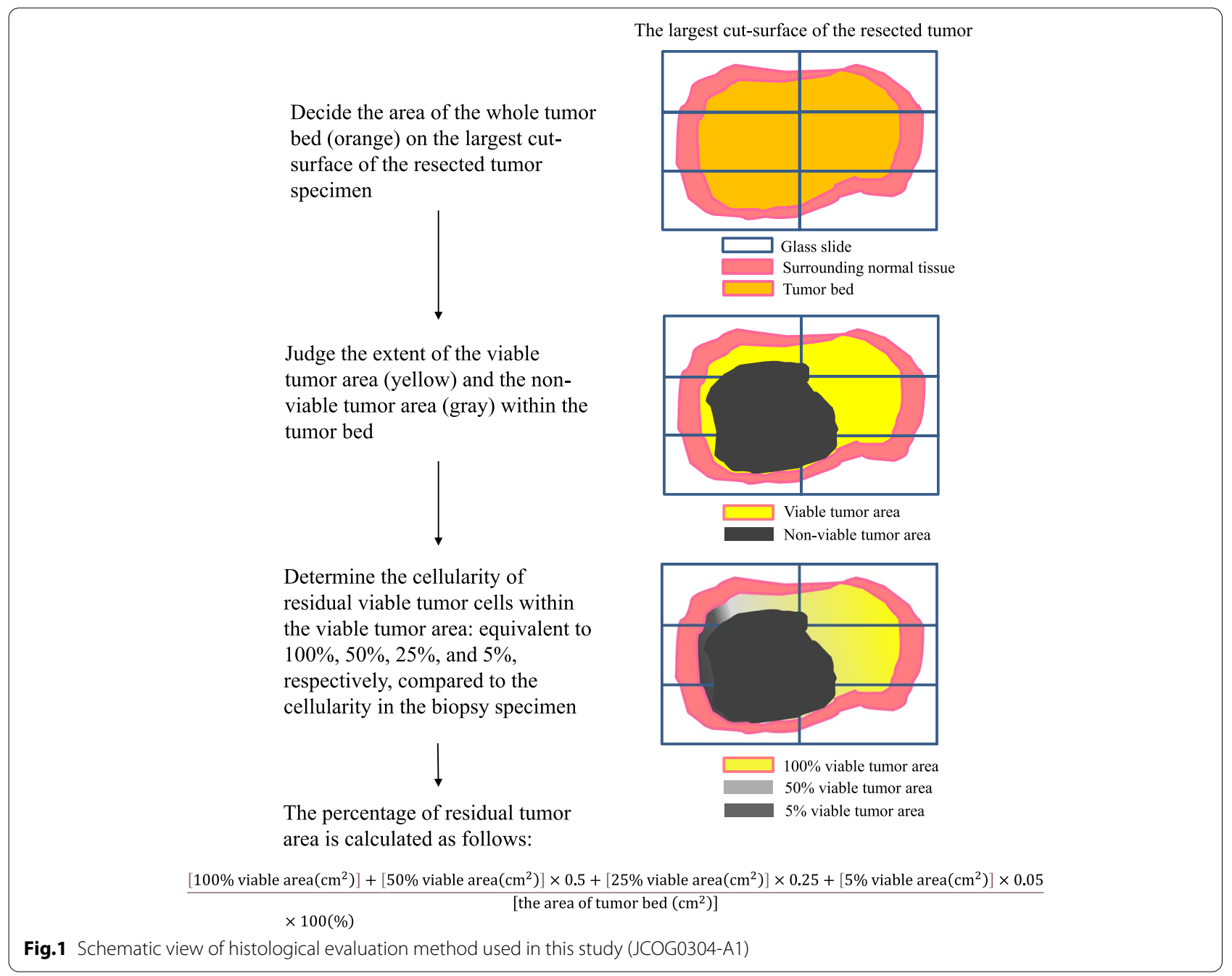

Overall survival (OS) was defined as the time from enrollment to death from any cause and censored at the date of last contact for a surviving patient. The OS was estimated using Kaplan-Meier method. Univariable analysis was performed to investigate the impact on OS. Hazard ratios and $p$ values were derived using Cox regression model. Continuous variable for group comparison was performed by Wilcoxon-rank sum test. The JCOG Data Center performed the statistical analyses using SAS version 9.4 (SAS Institute, Cary, NC).

\section{Results}

\section{Patient characteristics}

From March 2004 to September 2008, 72 patients were enrolled into the JCOG0304 trial, and 64 of the 72 patients eligible for this accompanying study were included (Fig. 2). The characteristics of the included patients are listed in Table 1 . Briefly, 30 patients were males and 34 were females, and the median age of patients was 47.5 years old (range 21-66). The most frequent tumor location was the thigh in 32 patients, followed by the buttock in 9 patients, the lower leg in 7 patients, the shoulder in 5 patients, the upper arm in 3 patients, the knee in 3 patients, the elbow in 2 patients, the forearm in 1 patient, the groin in 1 patient, and the ankle in 1 patient. The median tumor size was $7.4 \mathrm{~cm}$. The histological diagnosis of tumors was as follows: undifferentiated pleomorphic sarcoma in 20 patients, synovial sarcoma in 17 patients, leiomyosarcoma in 12 patients, myxofibrosarcoma in 7 patients, pleomorphic liposarcoma in 3 patients, myxoid liposarcoma in 2 patients, undifferentiated/unclassified sarcoma in 2 patients, and fibrosarcoma in 1 patient.

\section{Histological findings}

The representative histological findings of reviewed specimens are shown in Fig. 3. The tumor specimens after chemotherapy were distinguishable into three 


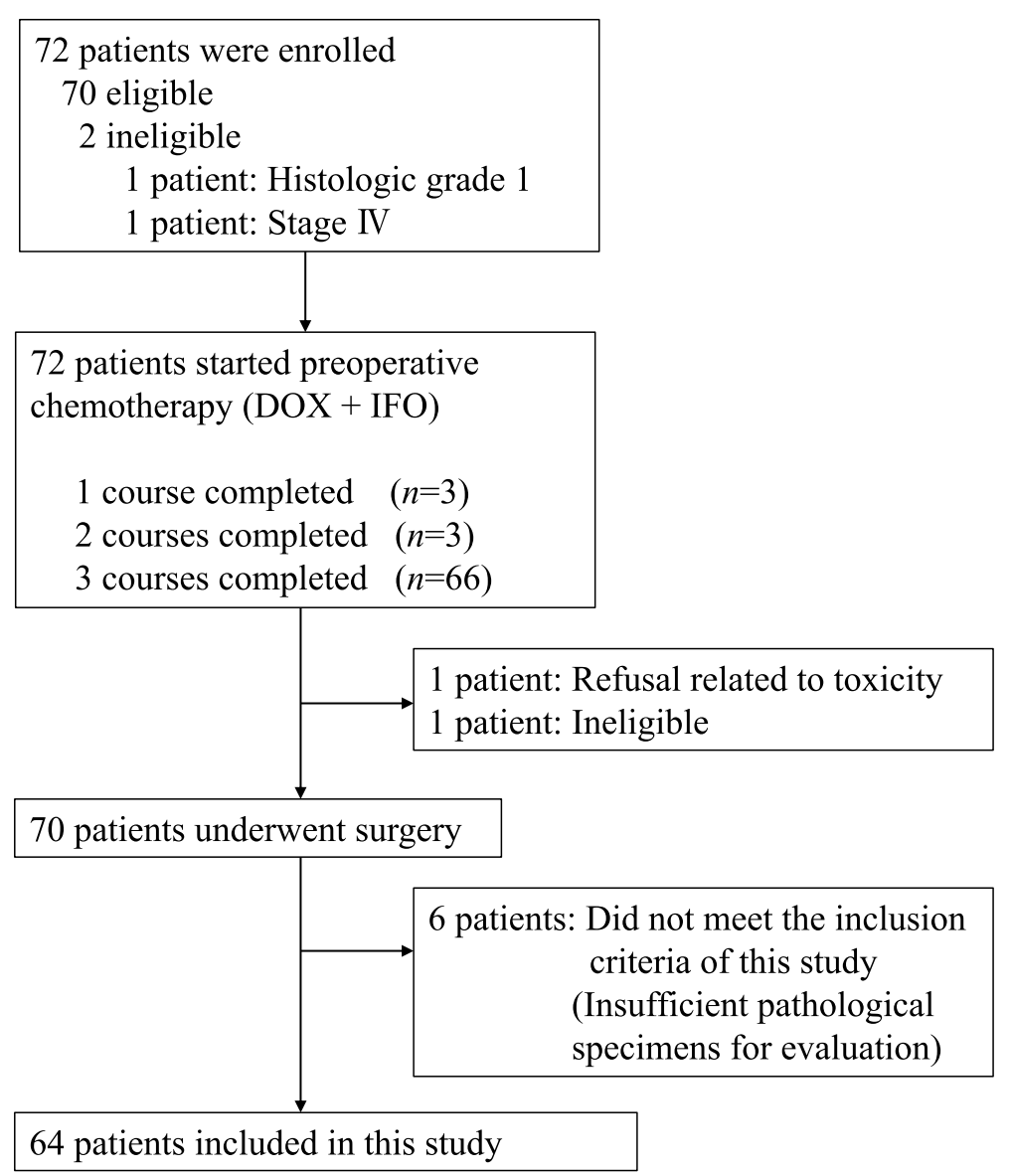

Fig.2 Patients flow diagram of this study (JCOG0304-A1)

histological areas: viable tumor area (Fig. 3a); necrotic area with irreversible nuclear changes (Fig. 3b, c, d); and degenerative area with stromal fibrosis, hyalinization, or cystic change (Fig. 3e, f). In all 64 assessed cases, the percentage of viable tumor area ranged from $0.1 \%$ to $97.0 \%$, with median value of $35.7 \%$.

\section{Concordance rate between pathologists}

In 10 cases of Group A, which was the test set group, the overall weighted $\kappa$ score was 0.73 . This value met the prespecified criteria of 0.6 and proceed to Group B. In Group $B$, which was the first validation set, the overall weighted $\kappa$ score was 0.84 . In the remaining 44 cases (Group C), the overall weighted $\kappa$ score was 0.64 . In total, the weighted $\kappa$ score in all patients was 0.72 .

\section{Association between histological response and radiological response}

Among 64 patients included in this accompanying study, $\mathrm{PR}$ in $14, \mathrm{SD}$ in 47 , and $\mathrm{PD}$ in 3 patients were observed, respectively. As shown in Fig. 4, the median percentage of the residual tumor area was 14\% (interquartile range: $4 \%-33 \%$ ) in PR and $40 \%$ (interquartile range: $15 \%-71 \%$ ) in non-PR (SD and PD). The percentage of the residual tumor in PR was significantly lower than that in non-PR $(p=0.0130)$.

\section{Prognostic analysis}

A median follow-up period of all cases was 10.3 years, and the shortest follow-up for survivors was 5.7 years. The 3-, 5-, and 7-year OS for 64 eligible patients was 87.5\% (95\% confidence interval [CI], 76.6-93.5\%), 84.4\% (95\% CI, 72.9-91.3\%) and 82.8\% (95\% CI, 71.0-90.1\%), respectively.

When the cut-off value of the percentage of the residual tumor area was set between $24.565 \%$ (the largest value among the responders) and $25.150 \%$ (the smallest value among the non-responders), the $p$-value for the difference in overall survival showed the minimum value (Fig. 5). Hazard ratio of the non-responder $(n=39)$, whose percentage of the residual tumor was more than 
Table 1 Patient characteristics $(n=64)$

\begin{tabular}{|c|c|}
\hline Gender & \\
\hline Male/Female & $30 / 34$ \\
\hline Age & \\
\hline Median, years & 47.5 \\
\hline Range & $21-66$ \\
\hline ECOG & \\
\hline Performance status & \\
\hline $0 / 1$ & $45 / 19$ \\
\hline Histological grade (FNCLCC) & \\
\hline $2 / 3$ & $41 / 23$ \\
\hline Site & \\
\hline Shoulder & 5 \\
\hline Upper arm & 3 \\
\hline Elbow & 2 \\
\hline Forearm & 1 \\
\hline Buttock & 9 \\
\hline Groin & 1 \\
\hline Thigh & 32 \\
\hline Knee & 3 \\
\hline Lower leg & 7 \\
\hline Ankle & 1 \\
\hline Tumor size, major axis (cm) & \\
\hline Median & 7.4 \\
\hline Range & $3.0-26.0$ \\
\hline $10 \mathrm{~cm}$ or less/More than $10 \mathrm{~cm}$ & $53 / 11$ \\
\hline Tumor size, minor axis $(\mathrm{cm})$ & \\
\hline Median & 5.3 \\
\hline Range & $1.6-13.0$ \\
\hline Histologicacl subtype (central review) & \\
\hline Undifferentiated pleomorphic sarcoma & 20 \\
\hline Synovial sarcoma & 17 \\
\hline Leiomyosarcoma & 12 \\
\hline Myxofibrosarcoma & 7 \\
\hline Pleomorphic liposarcoma & 3 \\
\hline Myxoid liposarcoma & 2 \\
\hline Undifferentiated/unclassified sarcoma & 2 \\
\hline Fibrosarcoma & 1 \\
\hline ECOG, Eastern Cooperative Oncology Group & \\
\hline
\end{tabular}

$25 \%$, to the responder $(n=25)$ was 4.029 (95\% CI, $0.893-$ $18.188, p=0.070$ ).

\section{Discussion}

In this study, we developed a standardized evaluation method of pathological response to preoperative chemotherapy in STS patients, which showed a substantial agreement and reproducibility score among six pathologists. The pathological response calculated here was associated with radiological response of PR or non-PR.
Patients with less than $25 \%$ of the residual viable tumor area showed a trend of better OS while the prognostic results were far from conclusive.

A thorough review of the previous papers on histological response to preoperative treatment and its prognostic significance in STSs revealed non-negligible differences in regimen of preoperative chemotherapy, combination use of radiotherapy, and evaluation method of histological response (Table 2). Most of the previous studies used retrospectively collected data and small sample size. Here, we present results of a prospective analysis performed as an accompanying study of a phase II trial (JCOG0304). Compared with the previous papers, the strengths of this study (JCOG0304A1) are 1) prospective data collection, 2) long follow-up duration with a median follow-up period of over 10 years, 3) the same preoperative treatment method specified in the protocol, and 4) a central pathology review using the standardized evaluation criteria. The radiological, histological, and prognostic data used in this study were of a sufficiently high quality to deliver a critical analysis, although sample size was not large enough to conclude the prognostic significance.

Standardization of evaluation method to assess histological response to chemotherapy in STSs was one of the purposes of this study. As many as six experienced pathologists met together at a central pathology review repeatedly to establish reliable evaluation criteria. We decided to count the residual viable tumor area but not necrotic area to assess the histological response to chemotherapy because discriminating between viable and degenerative area was considered easier than distinguishing between necrotic and degenerative area. As a result, we could design the evaluation method that showed substantial concordance rate among pathologists. As for osteosarcoma, histological response to preoperative chemotherapy has been used more widely for risk stratification than STSs [25]. The most popular cut-off value between good and poor responses in osteosarcoma is $90 \%$ in necrosis, although some papers suggested the need for re-evaluation [26]. The evaluation method established in this study will be used as one of the standard procedures for the assessment of histological response to preoperative chemotherapy in STSs, and could be applied to osteosarcoma in the future research.

Our analysis indicated that the cut-off value of $25 \%$ of residual viable tumor cells showed the closest correlation with the prognosis, although the association was not statistically significant $(p=0.070)$. Our results were closest to those of Issels's report in which the cut-off value of necrosis was set to $75 \%$ [13]. The cut-off value varied greatly among the previous papers listed in Table 2 . As stated earlier, the process for setting the cut-off value 

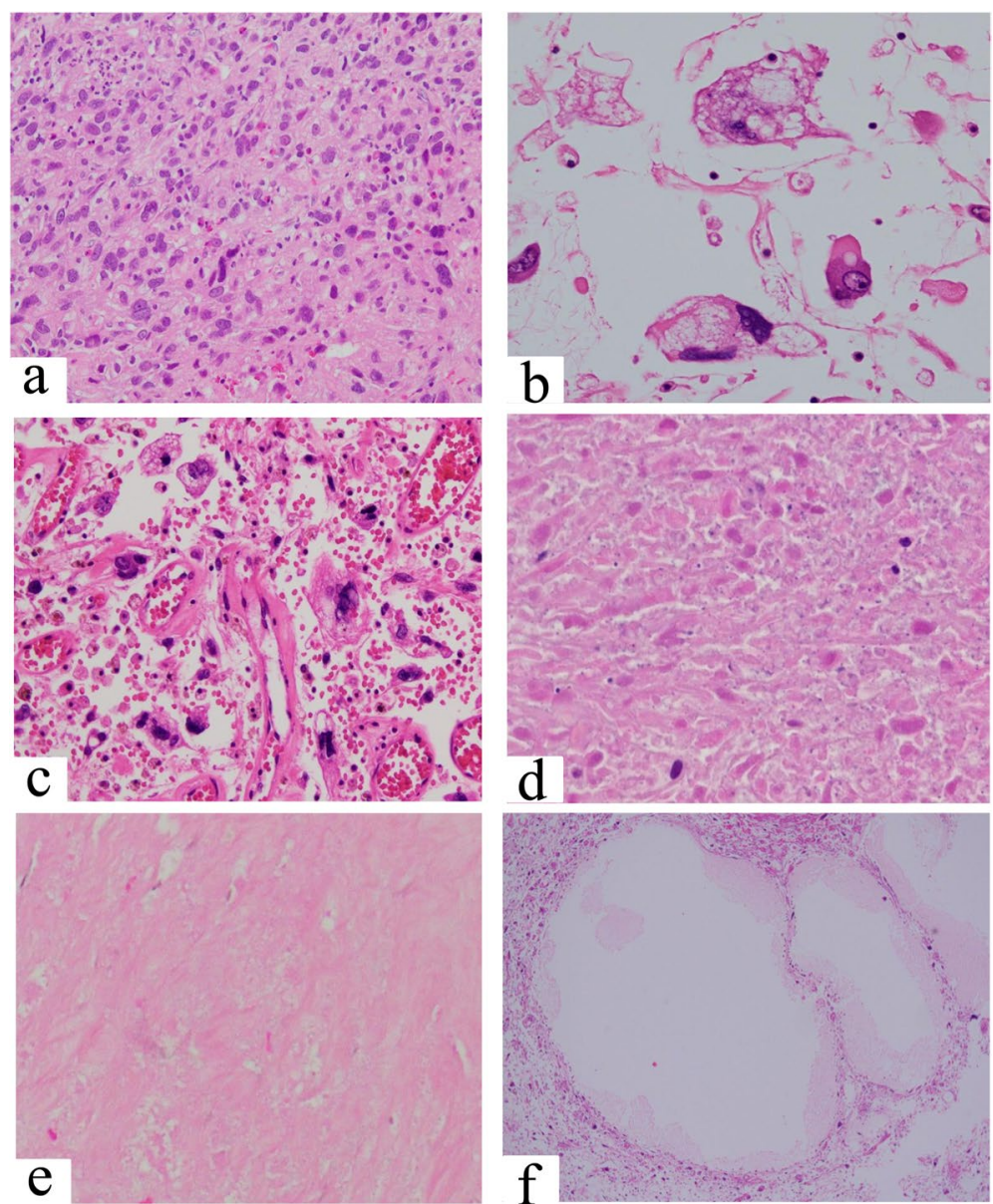

Fig.3 Representative histological appearance treated with preoperative chemotherapy (Hematoxylin and eosin stain, original magnification $\times 400$ ): a. Viable tumor cells with slight nuclear swelling. b. Non-viable tumor cells showing degenerative change. The tumor cells changed into large, bizarre and multinucleated cells with vacuolated chromatin. c. Non-viable tumor cells with pyknosis, karyorrhexis and karyolysis. d. Necrotic tumor cells with loss of nuclear staining. e. fibrosis and hyalinized stroma. f. Intratumoral cystic change

has not been clearly established in previous studies. We believe that the differences in regimen of preoperative chemotherapy, concurrent therapy, included histological diagnosis, and histological evaluation method may be accountable for the differences in the cut-off value. Especially in STS, the radiological response to preoperative chemotherapy is not reliable prognostic predictor. In JCOG0304, the radiological response demonstrated no association with survival of patients with operable soft tissue sarcoma [8]. It is particularly worth noting that histological responders in our study showed an excellent prognosis, achieving up to $92.0 \%$ of 10 -year OS. Prognostic prediction with our histological evaluation method could be a useful tool in the clinical management of STS patients who received preoperative chemotherapy with DOX and IFO.

There are some limitations to this study, which include technical difficulties in assessing viability of the tumor cells, presence of intratumoral necrosis prior to chemotherapy, heterogeneity of histology, and sample size. Moreover, technically, there are marginal histological differences in appearances of viable and non-viable tumor cells making it difficult to distinguish between the two. Therefore, decisions on evaluation of the tumor cell viability after severe degenerative damage caused by chemotherapeutic agents are sometimes split among pathologists. In our experience, a central pathology review helped pathologists to lower the discrepancy and to share common understandings of the pathological features. In the future, by means of distribution of plenty of histological pictures of JCOG0304A1 cases, the evaluation criteria established here should be disseminated to the pathologists who engage in the diagnosis of STS. STSs occasionally have intratumoral necrosis and hemorrhage in nature. It is therefore difficult to differentiate between necrosis caused by chemotherapy and the preexisting one. STSs comprise of a variety of histological entities. Histology may affect the pathological response 


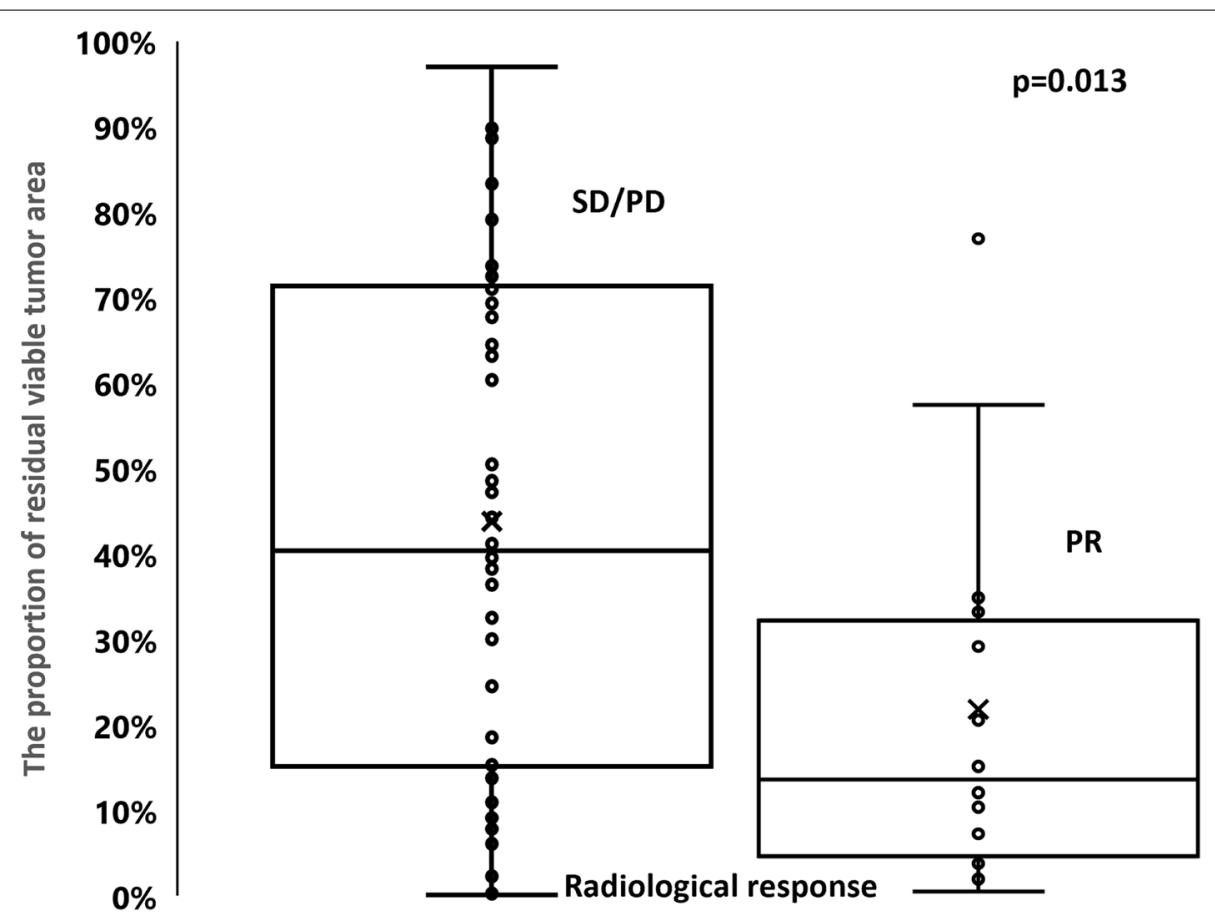

Fig.4 Association between histological response and radiological response. Among 64 patients included in this study, PR, SD, and PD were observed in 14,47, and 3 patients, respectively. The median percentage of the residual tumor area was $14 \%$ in PR and 40\% in non-PR (SD and PD). The percentage of the residual tumor in PR was significantly lower than that in non-PR ( $p=0.0130)$. * PR: partial response; SD: stable disease; PD: progressive disease

\section{Kaplan-Meier estimate of overall survival by histological response}

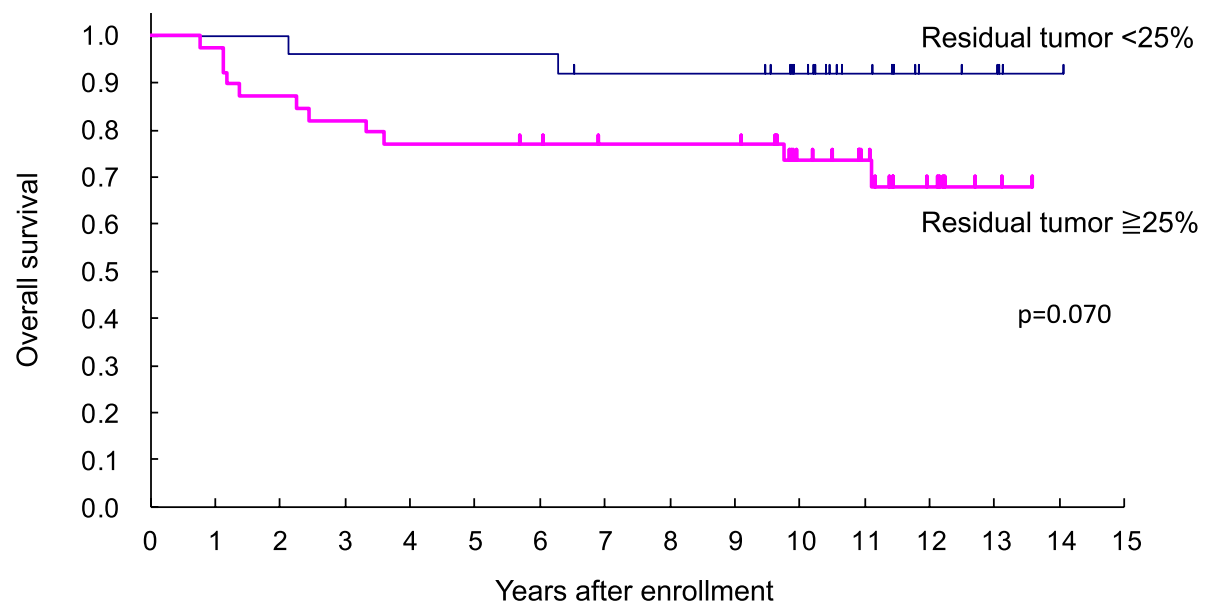

Fig.5 Kaplan-Meier estimate of overall survival by histological response. When the cut-off value of the percentage of the residual tumor area was set as $25 \%$, the $p$-value for the difference in OS showed the minimum value. Hazard ratio of the non-responder $(n=39)$, whose percentage of the residual tumor was more than $25 \%$, to the responder $(n=25)$ was $4.029(95 \% \mathrm{Cl}, 0.893-18.188, p=0.070)$

analyzed in this study. A total of seven different kinds of STS were included in this study. Finally, the number of the patients included in this study $(n=64)$ was not high enough to perform prognostic evaluation including multivariate analysis, even though we had a long followup duration and a high-quality data. We believe that further investigations with more patients are necessary to draw a more accurate conclusion. 
Oda et al. BMC Cancer $\quad$ (2022) 22:94

Page 9 of 12

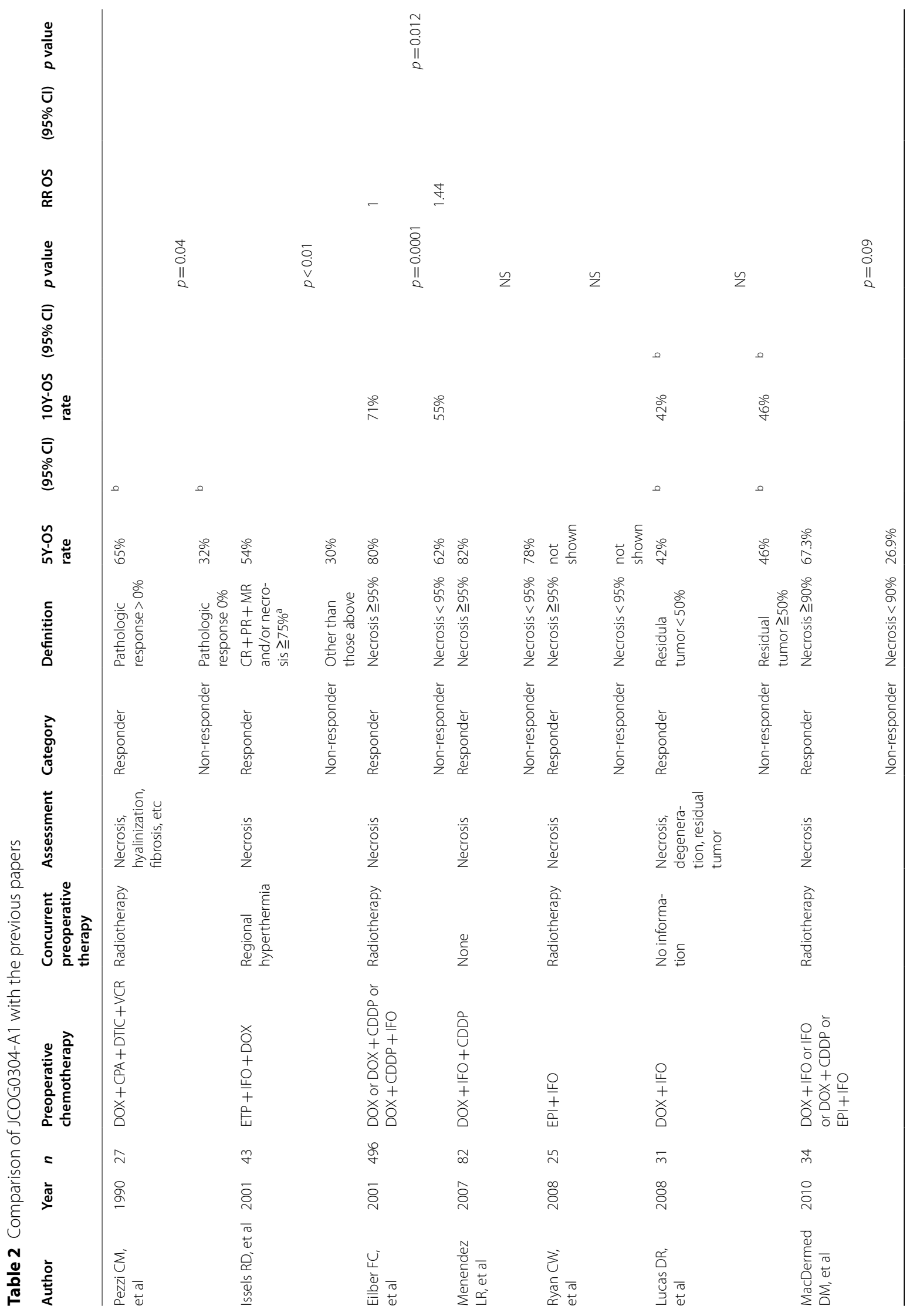




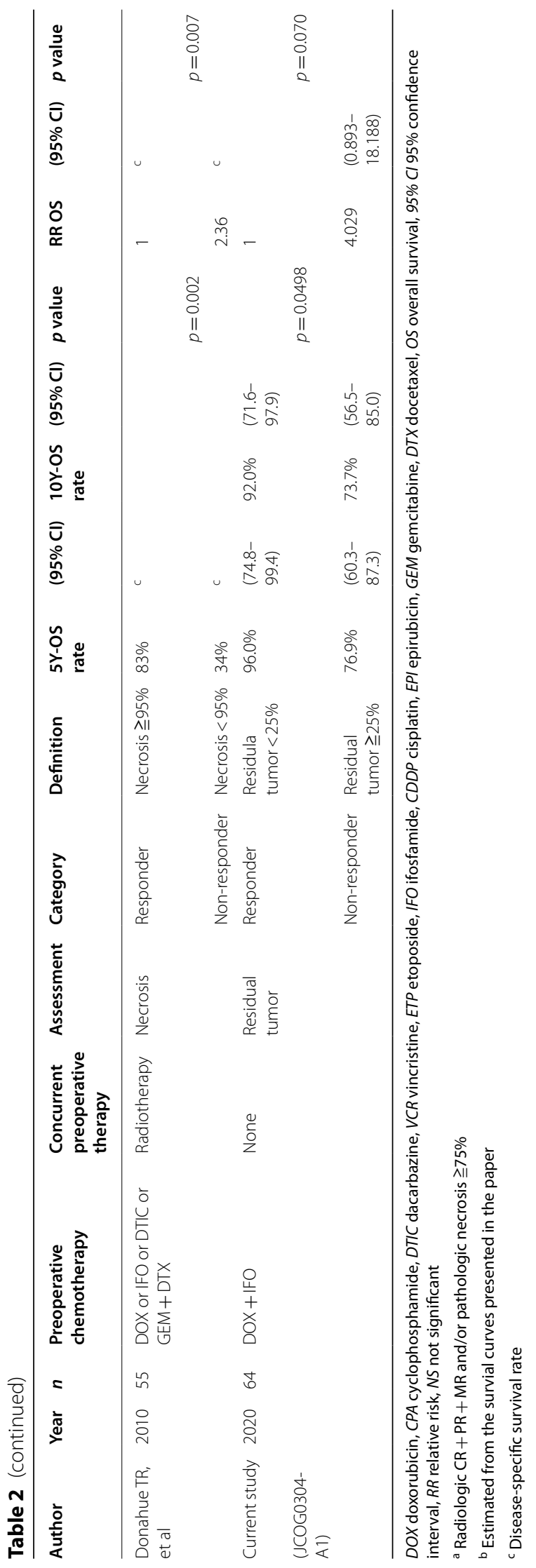




\section{Conclusions}

In summary, the JCOG0304-A1 evaluation method of pathological response to preoperative chemotherapy in STSs showed a substantial agreement and reproducibility score. Patients with over $25 \%$ of the residual viable tumor area tended to show better OS than others in this study. The evaluation method established here was useful for estimating the prognosis of STS patients administered perioperative chemotherapy with doxorubicin and ifosfamide.

\begin{abstract}
Abbreviations
JCOG: Japan Clinical Oncology Group; STS: Soft tissue sarcoma; DOX: Doxorubicin; IFO: Ifosfamide; AJCC: American Joint Committee on Cancer; UICC: International Union against Cancer; FNCLCC: Federation Nationale des Centres de Lutte le Cancer; MRI: Magnetic resonance imaging; WHO: World Health Organization; CR: Complete response; PR: Partial response; SD: Stable disease; PD: Progressive disease; OS: Overall survival; Cl: Confidence interval.
\end{abstract}

\section{Acknowledgements}

We would like to thank all members of the JCOG Bone and Soft Tissue Tumor Study Group. Participating Institutions of JCOG0304-A1 were as follows (from north to south): Sapporo Medical University, Hokkaido Cancer Center, Tohoku University, Chiba Cancer Center, National Cancer Center Hospital, Nihon University, Kyorin University, Keio University, Teikyo University, Kanagawa Cancer Center, Niigata Cancer Center Hospital, Kanazawa University, Gifu University, Shizuoka Cancer Center, Mie University, Kyoto University, Osaka International Cancer Center, Okayama University, Kyushu University, National Kyushu Cancer Center. We would like to thank Editage (www.editage.com) for English language editing.

\section{Authors' contributions}

Conceptualization: Yoshinao Oda, Kazuhiro Tanaka, Takeaki Ishii, Makoto Endo, Junki Mizusawa, Haruhiko Fukuda; Protocol development: Yoshinao Oda, Takeaki Ishii, Makoto Endo, Junki Mizusawa, Haruhiko Fukuda; Data collection: Yoshinao Oda, Kazuhiro Tanaka, Takanori Hirose, Tadashi Hasegawa, Nobuyuki Hiruta, Masanori Hisaoka, Masato Yoshimoto, Hiroshi Otsuka, Hirofumi Bekki, Takeaki Ishii, Makoto Endo, Toshiyuki Kunisada, Toru Hiruma, Hiroyuki Tsuchiya, Hirohisa Katagiri, Yoshihiro Matsumoto, Akira Kawai, Robert Nakayama, Hiroyuki Kawashima, Satoshi Takenaka, Makoto Emori, Munenori Watanuki, Yukihiro Yoshida, Takeshi Okamoto, Toshifumi Ozaki, Yukihide Iwamoto, Takayuki Nojima; Data analysis: Junki Mizusawa, Haruhiko Fukuda; Data interpretation: Yoshinao Oda, Kazuhiro Tanaka, Takanori Hirose, Tadashi Hasegawa, Nobuyuki Hiruta, Masanori Hisaoka, Masato Yoshimoto, Makoto Endo, Junki Mizusawa, Haruhiko Fukuda; Writing —original draft preparation: Yoshinao Oda, Masato Yoshimoto, Makoto Endo; Writing — review and editing: Kazuhiro Tanaka, Takanori Hirose, Tadashi Hasegawa, Nobuyuki Hiruta, Masanori Hisaoka, Hiroshi Otsuka, Hirofumi Bekki, Takeaki Ishii, Toshiyuki Kunisada, Toru Hiruma, Hiroyuki Tsuchiya, Hirohisa Katagiri, Yoshihiro Matsumoto, Akira Kawai, Robert Nakayama, Hiroyuki Kawashima, Satoshi Takenaka, Makoto Emori, Munenori Watanuki, Yukihiro Yoshida, Takeshi Okamoto, Junki Mizusawa, Haruhiko Fukuda, Toshifumi Ozaki, Yukihide Iwamoto, Takayuki Nojima; Funding acquisition: Yoshinao Oda, Kazuhiro Tanaka, Toshifumi Ozaki, Yukihide Iwamoto; Supervision: Kazuhiro Tanaka, Toshifumi Ozaki, Yukihide Iwamoto, Takayuki Nojima; Approval of final manuscript: All authors.

\section{Funding}

This work was supported in part by the National Cancer Center Research and Development Fund [grant number 29-A-3 and 2020-J-3], JSPS KAKENHI [grant number JP19H03444] and AMED [grant number JP17ck0106336 and JP20ck0106614] for the collection, analysis, and interpretation of data and for preparing the manuscript.

\section{Availability of data and materials}

The datasets generated and/or analyzed during the current study are not publicly available due to rules of the "Japanese Ethical Guidelines for Medical and Health Research Involving Human Subjects" but are available from the corresponding author on reasonable request.

\section{Declarations}

\section{Competing interests}

Akira Kawai reports personal fees from Takara-Bio, Daiichi Sankyo, Eli Lilly, Eisai, Novartis and Taiho Pharmaceutical outside the submitted work. Makoto Endo reports personal fees from Taisho Pharmaceutical, Daiichi Sankyo, Eli Lilly, Eisai, Novartis, Ayumi Pharmaceutical, and Taiho Pharmaceutical outside the submitted work. Junki Mizusawa reports grants from Ministry of Health, Labour and Welfare, Japan during the conduct of the study; personal fee from Chuugai Pharmaceutical outside the submitted work. Haruhiko Fukuda reports lecture fee from Chuugai Pharmaceutical outside the submitted work. The other authors have nothing to disclose.

\section{Ethics approval and consent to participate}

All methods were carried out in accordance with relevant guidelines and regulations. This study (the Japan Clinical Oncology Group study, JCOG0304A1) protocol was approved by the Protocol Review Committee of the JCOG and by the Institutional Review Boards in each of the 20 participating institutes. Informed consent to participate and publication was obtained from all the patients who were enrolled in the JCOG0304 trial.

\section{Consent for publication}

Consent for publication was obtained by all authors prior to submission.

\section{Conflict of interest}

Akira Kawai reports personal fees from Takara-Bio, Daiichi Sankyo, Eli Lilly, Eisai, Novartis and Taiho Pharmaceutical outside the submitted work. Makoto Endo reports personal fees from Taisho Pharmaceutical, Daiichi Sankyo, Eli Lilly, Eisai, Novartis, Ayumi Pharmaceutical, and Taiho Pharmaceutical outside the submitted work. Junki Mizusawa reports grants from Ministry of Health, Labour and Welfare, Japan during the conduct of the study; personal fee from Chuugai Pharmaceutical outside the submitted work. Haruhiko Fukuda reports lecture fee from Chuugai Pharmaceutical outside the submitted work. The other authors have nothing to disclose.

\section{Author details}

${ }^{1}$ Department of Anatomic Pathology, Graduate School of Medical Sciences, Kyushu University, Fukuoka, Japan. ${ }^{2}$ Department of Orthopaedic Surgery, Faculty of Medicine, Oita University, Oita, Japan. ${ }^{3}$ Department of Diagnostic Pathology, Hyogo Cancer Center, Akashi, Japan. ${ }^{4}$ Department of Surgical Pathology, School of Medicine, Sapporo Medical University, Sapporo, Japan. ${ }^{5}$ Department of Surgical Pathology, Toho University Sakura Medical Center, Sakura, Japan. ${ }^{6}$ Department of Pathology and Oncology, School of Medicine, University of Occupational and Environmental Health, Kitakyushu, Japan. ${ }^{7}$ Department of Orthopaedic Surgery, Graduate School of Medical Sciences, Kyushu University, Fukuoka, Japan. ${ }^{8}$ Department of Medical Materials for Musculoskeletal Reconstruction, Okayama University Graduate School of Medicine, Dentistry, and Pharmaceutical Sciences, Okayama, Japan. ${ }^{9}$ Department of Musculoskeletal Tumor Surgery, Kanagawa Cancer Center, Yokohama, Japan. ${ }^{10}$ Department of Orthopaedic Surgery, Graduate School of Medical Sciences, Kanazawa University, Kanazawa, Japan. ${ }^{11}$ Division of Orthopaedic Oncology, Shizuoka Cancer Center Hospital, Shizuoka, Japan. ${ }^{12}$ Department of Musculoskeletal Oncology, National Cancer Center Hospital, Tokyo, Japan. ${ }^{13}$ Department of Orthopaedic Surgery, Keio University School of Medicine, Tokyo, Japan. ${ }^{14}$ Division of Orthopedic Surgery, Department of Regenerative and Transplant Medicine, Niigata University Graduate School of Medical and Dental Sciences, Niigata, Japan. ${ }^{15}$ Department of Orthopaedic Surgery, Osaka International Cancer Institute, Osaka, Japan. ${ }^{16}$ Department of Orthopaedic Surgery, Sapporo Medical University School of Medicine, Sapporo, Japan. ${ }^{17}$ Department of Orthopaedic Surgery, Tohoku University School of Medicine, 
Sendai, Japan. ${ }^{18}$ Department of Orthopaedic Surgery, Nihon University Itabashi Hospital, Tokyo, Japan. ${ }^{19}$ Department of Orthopaedic Surgery, Kyoto University Graduate School of Medicine, Kyoto, Japan. ${ }^{20} \mathrm{JCOG}$ Data Center, National Cancer Center Hospital, Tokyo, Japan. ${ }^{21}$ Department of Orthopaedic Surgery, Okayama University Graduate School of Medicine, Dentistry, and Pharmaceutical Sciences, Okayama, Japan. ${ }^{22}$ Department of Orthopaedic Surgery, Kyushu Rosai Hospital, Kitakyushu, Japan. ${ }^{23}$ Department of Pathology, Graduate School of Medical Sciences, Kanazawa University, Kanazawa, Japan.

Received: 2 February 2021 Accepted: 12 January 2022

Published online: 21 January 2022

\section{References}

1. Siegel RL, Miller KD, Jemal A. Cancer statistics, 2020. CA Cancer J Clin. 2020;70(1):7-30.

2. Amin MB, Edge SB, Greene FL, Byrd DR, Brookland RK, Washington MK, Gershenwald JE, Compton CC, Hess KR, Sullivan DC et al (eds.): The AJCC Cancer Staging Manual, 8th edn. Switzerland: Springer Nature; 2017.

3. Pervaiz N, Colterjohn N, Farrokhyar F, Tozer R, Figueredo A, Ghert M. A systematic meta-analysis of randomized controlled trials of adjuvant chemotherapy for localized resectable soft-tissue sarcoma. Cancer. 2008:113(3):573-81.

4. Gronchi A, Ferrari S, Quagliuolo V, Broto JM, Pousa AL, Grignani G, Basso U, Blay JY, Tendero O, Beveridge RD, et al. Histotype-tailored neoadjuvant chemotherapy versus standard chemotherapy in patients with high-risk soft-tissue sarcomas (ISG-STS 1001): an international, openlabel, randomised, controlled, phase 3, multicentre trial. Lancet Oncol. 2017;18(6):812-22.

5. Gronchi A, Palmerini E, Quagliuolo V, Martin Broto J, Lopez Pousa A, Grignani G, Brunello A, Blay JY, Tendero O, Diaz Beveridge R, et al. Neoadjuvant Chemotherapy in High-Risk Soft Tissue Sarcomas: Final Results of a Randomized Trial From Italian (ISG), Spanish (GEIS), French (FSG), and Polish (PSG) Sarcoma Groups. J Clin Oncol. 2020;38(19):2178-86.

6. Tanaka K, Mizusawa J, Fukuda H, Araki N, Chuman H, Takahashi M, Ozaki T, Hiruma T, Tsuchiya H, Morioka H, et al. Perioperative chemotherapy with ifosfamide and doxorubicin for high-grade soft tissue sarcomas in the extremities (JCOG0304). Jpn J Clin Oncol. 2015;45(6):555-61.

7. Tanaka K, Mizusawa J, Naka N, Kawai A, Katagiri H, Hiruma T, Matsumoto Y, Tsuchiya H, Nakayama R, Hatano H, et al. Ten-year follow-up results of perioperative chemotherapy with doxorubicin and ifosfamide for highgrade soft-tissue sarcoma of the extremities: Japan Clinical Oncology Group study JCOG0304. BMC Cancer. 2019;19(1):890.

8. Tanaka K, Ogawa G, Mizusawa J, Naka N, Kawai A, Takahashi M, Hiruma T, Matsumoto Y, Tsuchiya $\mathrm{H}$, Nakayama R, et al. Prospective comparison of various radiological response criteria and pathological response to preoperative chemotherapy and survival in operable high-grade soft tissue sarcomas in the Japan Clinical Oncology Group study JCOG0304. World J Surg Oncol. 2018;16(1):162.

9. Ueda T, Naka N, Araki N, Ishii T, Tsuchiya H, Yoshikawa H, Mochizuki K, Tsuboyama T, Toguchida J, Ozaki T, et al. Validation of radiographic response evaluation criteria of preoperative chemotherapy for bone and soft tissue sarcomas: Japanese Orthopaedic Association Committee on Musculoskeletal Tumors Cooperative Study. J Orthop Sci. 2008;13(4):304-12.

10. Donahue TR, Kattan MW, Nelson SD, Tap WD, Eilber FR, Eilber FC. Evaluation of neoadjuvant therapy and histopathologic response in primary, high-grade retroperitoneal sarcomas using the sarcoma nomogram. Cancer. 2010;116(16):3883-91.

11. Eilber FC, Rosen G, Eckardt J, Forscher C, Nelson SD, Selch M, Dorey F, Eilber FR. Treatment-induced pathologic necrosis: a predictor of local recurrence and survival in patients receiving neoadjuvant therapy for highgrade extremity soft tissue sarcomas. J Clin Oncol. 2001;19(13):3203-9.

12. Henshaw RM, Priebat DA, Perry DJ, Shmookler BM, Malawer MM. Survival after induction chemotherapy and surgical resection for high-grade soft tissue sarcoma. Is radiation necessary? Ann Surg Oncol. 2001;8(6):484-95.
13. Issels RD, Abdel-Rahman S, Wendtner C, Falk MH, Kurze V, Sauer H, Aydemir U, Hiddemann W. Neoadjuvant chemotherapy combined with regional hyperthermia (RHT) for locally advanced primary or recurrent high-risk adult soft-tissue sarcomas (STS) of adults: long-term results of a phase II study. Eur J Cancer. 2001;37(13):1599-608.

14. Lucas DR, Kshirsagar MP, Biermann JS, Hamre MR, Thomas DG, Schuetze SM, Baker LH. Histologic alterations from neoadjuvant chemotherapy in high-grade extremity soft tissue sarcoma: clinicopathological correlation. Oncologist. 2008;13(4):451-8.

15. MacDermed DM, Miller LL, Peabody TD, Simon MA, Luu HH, Haydon RC, Montag AG, Undevia SD, Connell PP. Primary tumor necrosis predicts distant control in locally advanced soft-tissue sarcomas after preoperative concurrent chemoradiotherapy. Int J Radiat Oncol Biol Phys. 2010;76(4):1147-53.

16. Menendez LR, AhImann ER, Savage K, Cluck M, Fedenko AN. Tumor necrosis has no prognostic value in neoadjuvant chemotherapy for soft tissue sarcoma. Clin Orthop Relat Res. 2007;455:219-24.

17. Pezzi CM, Pollock RE, Evans HL, Lorigan JG, Pezzi TA, Benjamin RS, Romsdahl MM. Preoperative chemotherapy for soft-tissue sarcomas of the extremities. Ann Surg. 1990;211(4):476-81.

18. Ryan CW, Montag AG, Hosenpud JR, Samuels B, Hayden JB, Hung AY, Mansoor A, Peabody TD, Mundt AJ, Undevia S. Histologic response of dose-intense chemotherapy with preoperative hypofractionated radiotherapy for patients with high-risk soft tissue sarcomas. Cancer. 2008;112(11):2432-9.

19. Tanaka K, Kawamoto H, Saito I, Yoshimura K, Fukuda H, Iwamoto Y. Preoperative and postoperative chemotherapy with ifosfamide and adriamycin for adult high-grade soft-tissue sarcomas in the extremities: Japan Clinical Oncology Group Study JCOG0304. Jpn J Clin Oncol. 2009;39(4):271-3.

20. Guillou L, Coindre JM, Bonichon F, Nguyen BB, Terrier P, Collin F, Vilain MO, Mandard AM, Le Doussal V, Leroux A, et al. Comparative study of the National Cancer Institute and French Federation of Cancer Centers Sarcoma Group grading systems in a population of 410 adult patients with soft tissue sarcoma. J Clin Oncol. 1997;15(1):350-62.

21. Therasse P, Arbuck SG, Eisenhauer EA, Wanders J, Kaplan RS, Rubinstein L, Verweij J, Van Glabbeke M, van Oosterom AT, Christian MC, et al. New guidelines to evaluate the response to treatment in solid tumors. European Organization for Research and Treatment of Cancer, National Cancer Institute of the United States, National Cancer Institute of Canada. J Natl Cancer Inst. 2000;92(3):205-16.

22. Miller $A B$, Hoogstraten $B$, Staquet $M$, Winkler $A$. Reporting results of cancer treatment. Cancer. 1981;47(1):207-14.

23. Mielke PW Jr, Berry KJ, Johnston JE. The exact variance of weighted kappa with multiple raters. Psychol Rep. 2007;101 (2):655-60.

24. Mielke PW Jr, Berry KJ, Johnston JE. Resampling probability values for weighted kappa with multiple raters. Psychol Rep. 2008;102(2):606-13.

25. Whelan JS, Bielack SS, Marina N, Smeland S, Jovic G, Hook JM, Krailo M, Anninga J, Butterfass-Bahloul T, Bohling T, et al. EURAMOS-1, an international randomised study for osteosarcoma: results from pre-randomisation treatment. Ann Oncol. 2015;26(2):407-14.

26. Tsuda Y, Tsoi K, Parry MC, Stevenson JD, Fujiwara T, Sumathi V, Jeys LM Impact of chemotherapy-induced necrosis on event-free and overall survival after preoperative MAP chemotherapy in patients with primary high-grade localized osteosarcoma. Bone Joint J. 2020;102-B(6):795-803.

\section{Publisher's Note}

Springer Nature remains neutral with regard to jurisdictional claims in published maps and institutional affiliations. 